\title{
Differential effects of habitual chow-based and semi-purified diets on lipid metabolism in lactating rats and their offspring
}

\author{
Josep Maria del Bas ${ }^{1}$, Antoni Caimari ${ }^{1 *}$, Enzo Ceresi ${ }^{2}$, Anna Arola-Arnal $^{3}$, Andreu Palou ${ }^{2}$, Lluís Arola ${ }^{1,3}$ \\ and Anna Crescenti ${ }^{1}$ \\ ${ }^{1}$ Centre Tecnològic de Nutrició i Salut (CTNS), TECNIO, CEICS, Avinguda Universitat, 1, 43204 Reus, Spain \\ ${ }^{2}$ Laboratory of Molecular Biology, Nutrition and Biotechnology, Universitat de les Illes Balears and CIBER de Fisiopatologia \\ de la Obesidad y Nutrición (CIBERobn), Palma de Mallorca, Spain \\ ${ }^{3}$ Departament de Bioquímica i Biotecnologia, Nutrigenomics Research Group, Universitat Rovira i Virgili, Tarragona, Spain
}

(Submitted 17 February 2014 - Final revision received 17 October 2014 - Accepted 3 December 2014 - First published online 27 February 2015)

\begin{abstract}
Diet during pregnancy and lactation is a critical factor in relation to the health of dams and their offspring. Currently, control diets used in metabolic imprinting studies differ in composition and type, i.e. semi-purified diets (SD) or chow-based diets (ND). The aim of the present study was to determine whether two widely used control diets, a SD and a ND, that mainly differ in fat content $(5.08$ and $3.26 \%$, respectively) and its sources (soyabean oil for the SD and cereals and fish for the ND), fibre (6 and 15\%, respectively), and cholesterol (26 and $69 \mathrm{mg} / \mathrm{kg}$ diet, respectively) can influence the lipid metabolism of dams and their offspring. Wistar rats were fed either the SD or the ND during pregnancy and lactation. At weaning, SD-fed dams presented severe hepatic steatosis and increased levels of circulating TAG, NEFA and insulin. Importantly, the offspring presented an altered plasma lipid profile. In contrast, the ND allowed for a normal gestation and lactation process, and did not affect the metabolism of offspring. In parallel, virgin rats fed the SD showed no metabolic alterations. A higher intake of SFA and MUFA and a lower consumption of PUFA observed in SD-fed dams during the lactation period could contribute to explaining the observed effects. In conclusion, two different control diets produced very different outcomes in the lipid metabolism of lactating rats and their offspring. The present results highlight the importance of the assessment of the metabolic state of dams when interpreting the results of metabolic programming studies.
\end{abstract}

\section{Key words: Hepatic steatosis: Semi-purified diets: Chow: Lactation: Plasma lipid profiles: Offspring}

Diet is recognised as one of the major environmental factors influencing the development of the embryo and fetus, as well as maternal health ${ }^{(1,2)}$. Epidemiological and animal studies have shown that maternal undernutrition, overnutrition and malnutrition during pregnancy and/or lactation are implicated in predisposing offspring to an increased risk of developing obesity, hypertension, dyslipidaemia and impaired glucose metabolism ${ }^{(1,2)}$. For instance, in rats, moderate maternal dietary protein restriction during pregnancy is known to alter phenotypes in the offspring, which manifests as hypertension, dyslipidaemia and impaired glucose metabolism ${ }^{(3,4)}$. Moreover, there is evidence that maternal high-fat feeding results in the offspring having exacerbated adiposity and modified expression of key proteins involved in hepatic insulin sensitivity ${ }^{(2)}$.
Special attention has been paid to dietary sucrose in rodent models because several alterations have been ascribed to sucrose intake during pregnancy and lactation in dams and their offspring. Thus, maternal sucrose intake during pregnancy increases body weight, body fat, plasma TAG level ${ }^{(5)}$ and hepatic lipid synthesis related to alterations in carbohydrate metabolism ${ }^{(6)}$, and alters the distribution of cholesterol among lipoprotein fractions ${ }^{(7)}$ in the offspring of mice and rats. In pregnant and non-pregnant rats, dietary sucrose intake induced increased levels of plasma TAG and hepatic lipogenesis compared with the intake of dietary $\operatorname{starch}^{(8)}$. In another study, sucrose-fed dams presented higher triacylglycerolaemia and hepatic lipid levels compared with dextrin-fed dams ${ }^{(4)}$. Moreover, sucrose feeding increased insulin resistance and the rate of fatty acid synthesis in the

Abbreviations: AIN, American Institute of Nutrition; F-ND group, female offspring of lactating rats fed for 43 d with the chow-based diet; F-SD group, female offspring of lactating rats fed for $43 \mathrm{~d}$ with the semi-purified diet; GOT, glutamic-oxaloacetic transaminase; M-ND group, male offspring of lactating rats fed for $43 \mathrm{~d}$ with the chow-based diet; M-SD group, male offspring of lactating rats fed for $43 \mathrm{~d}$ with the semi-purified diet; ND, chow-based diet; ND-C group, control non-lactating group fed the chow-based diet; ND-L group, group fed the chow-based diet during pregnancy and lactation; SD, semi-purified diet; SD-C group, control non-lactating group fed the semi-purified diet; SD-L group, group fed the semi-purified diet during pregnancy and lactation. 
liver of dams and their respective fetuses ${ }^{(9)}$. Taken together, these studies highlight the importance of diet and, in particular, the amount of sucrose and lipids in the diet, in animal studies involving gestation and lactation, including experiments that intended to study the effect of maternal nutrition on their offspring.

Diets used in animal studies are prepared either from natural ingredients (chow-based diet; ND) or from more refined products (purified or semi-purified diet; SD). ND usually contain poorly specified ingredients that may strongly vary in composition between batches and between providers. For example, a 5000-fold difference in the level of phytooestrogens has been encountered ${ }^{(10)}$. This variability in ND, among other disadvantages, has led to increased use of SD in animal studies. These diets are made with refined ingredients, such as casein, maize starch, sucrose, maize oil, and a fixed amount of vitamins and minerals, and were designed to reduce the inherent variations in ND, allowing more reproducible experiments. The American Institute of Nutrition (AIN) promoted the use of nutritionally adequate purified diets for rodents. The first purified diet published by the AIN was the AIN-76 diet in 1976. Later, after some problems were associated with the AIN-76 formulation, this diet was replaced by the AIN-93 diet. One of the major changes introduced with this diet was the replacement of most of the sucrose by maize starch to avoid the possible adverse effects of sucrose. The recommended amount of sucrose for both AIN-93 diets was $100 \mathrm{~g} / \mathrm{kg}$ diet $^{(11)}$. Additionally, two different formulations were generated for the AIN-93 diet: an AIN-93G diet for growth, pregnancy and lactation phases, and an AIN-93M diet for the maintenance of adult rodents. The main difference between the two diets is that the AIN-93M diet has a lower protein and fat content than the AIN-93G diet ${ }^{(11)}$. Although there is a specific SD for gestating and growing rodents, there are numerous examples of studies in the literature that use a non-specific high-sucrose SD during these periods $^{(12-16)}$. Moreover, although, in recent years, many scientists have shifted to using SD, the use of ND is still common among animal studies designed to study the metabolic imprinting effects of nutritional interventions on dams and their offspring ${ }^{(17-23)}$. While there have been studies that use normal-fat ND for dams and their offspring ${ }^{(17-20)}$, other studies have used normal-fat ND for pregnant and lactating rats and SD for the offspring ${ }^{(21,22)}$. Importantly, many metabolic imprinting studies using $\mathrm{SD}^{(13-15,24,25)}$ and $\mathrm{ND}^{(17,19,21)}$ have focused on the offspring and failed to evaluate the status of the dams.

Taking into account that different scientific lines of evidence point to the detrimental effects of high sucrose intake on dams and their offspring, and keeping in mind that the effects of SD and ND on the health status of dams have been poorly evaluated in the context of metabolic imprinting studies, the elucidation and comparison of the impact of SD and ND with low sucrose content on the metabolism of dams could be of great interest. Therefore, the aim of the present study was to elucidate the effects of two normal-fat diets with low sucrose content, a SD and a ND, on lipid and glucose metabolism in rats during pregnancy and lactation and in their offspring. We chose a ND (A04; Panlab) and the D01060501 diet (Research Diets, Inc.), a SD based on the D12450B diet (Research Diets, Inc.) in which most of the sucrose was replaced by maize starch according to the guidelines of the AIN 93M diet. These diets have been widely used in research designed to study the metabolic imprinting effects of nutritional interventions ${ }^{(12-22,26)}$.

\section{Experimental methods}

\section{Ethics statement}

The experimental protocol was approved by the Animal Ethics Committee of the University Rovira i Virgili (Tarragona, Spain), following the 'Principles of laboratory animal care', and was carried out in accordance with the European Communities Council Directive (86/609/EEC).

\section{Animals}

The animals used in the present study were 12-week-old female virgin Wistar rats (Charles River Laboratories) weighing $210-220 \mathrm{~g}$. All the animals were housed at $22^{\circ} \mathrm{C}$ with a $12 \mathrm{~h}$ light-12 h dark cycle (lights on at 09.00 hours).

\section{Diets}

In the present study, two diets were used, a semi-purified diet (D01060501; Research Diets, Inc.) and a ND (A04; Panlab). In the SD, the main source of protein was casein. Maize starch and maltodextrin were the main contributors to the carbohydrate content, whereas cellulose was the fibre source. In contrast, the ND was prepared from natural ingredients. Proteins were mainly provided by fish, soyabean meal and yeast, and carbohydrates and fibre came from cereals, bran and middling. Concerning the lipid composition, although both diets can be considered as normal-fat diets, they differed in fat content $(3.26 \%$ in the ND and $5.08 \%$ in the SD) and its sources (cereals, such as maize grain and barley, fish and soyabean meal for the ND and lard and soyabean oil for the $\mathrm{SD})$. To have a comparable composition between the diets, the gross composition of both diets was analysed by a reference analytical laboratory (Laboratori Dr Vidal-general Lab SL), as shown in Table 1.

\section{Experimental design}

To achieve the objectives of the present study, a variety of experimental approaches were performed.

Lactation experiment. A total of eighteen female virgin rats were mated with males until impregnation (confirmed by the presence of sperm in vaginal smears). The day of impregnation was designated as day 0 of pregnancy. Afterwards, the rats were housed singly and divided into two groups ( $n$ ) ) depending on the type of diet received during pregnancy and lactation $(43-44 \mathrm{~d})$. One group was fed the ND (ND-L group) and the other group was fed the SD (SD-L group). Dams were fed ad libitum, and their body weight 
Table 1. Composition of the chow-based diet (ND) and semi-purified $\operatorname{diet}(\mathrm{SD})$

\begin{tabular}{lcc}
\hline & $\mathrm{ND}^{*}$ & $\mathrm{SD}^{*}$ \\
\hline Energy $(\mathrm{kcal} / 100 \mathrm{~g})$ & 299 & 367 \\
Energy $(\mathrm{kJ} / 100 \mathrm{~g})$ & 1251 & 1536 \\
Carbohydrate $(\mathrm{g} / \mathrm{kg})$ & 510 & 670 \\
Starch $(\mathrm{g} / \mathrm{kg})$ & 477 & 490 \\
Sucrose $(\mathrm{g} / \mathrm{kg})$ & $30 \cdot 3$ & $39 \cdot 6$ \\
Maltodextrin $(\mathrm{g} / \mathrm{kg})$ & 0 & 120 \\
Fat $(\mathrm{g} / \mathrm{kg})$ & $32 \cdot 6$ & $50 \cdot 8$ \\
SFA $(\mathrm{g} / \mathrm{kg})$ & $7 \cdot 60$ & $13 \cdot 2$ \\
Palmitic acid, $16: 0(\mathrm{~g} / \mathrm{kg}) \dagger$ & $6 \cdot 12$ & $8 \cdot 18$ \\
Stearic acid, $18: 0(\mathrm{~g} / \mathrm{kg}) \dagger$ & $0 \cdot 67$ & $3 \cdot 96$ \\
MUFA $(\mathrm{g} / \mathrm{kg})$ & $7 \cdot 20$ & $15 \cdot 2$ \\
Oleic acid, $18: 1(\mathrm{~g} / \mathrm{kg}) \dagger$ & $6 \cdot 03$ & $13 \cdot 9$ \\
PUFA $(\mathrm{g} / \mathrm{kg})$ & $17 \cdot 8$ & $22 \cdot 4$ \\
Linoleic acid, $18: 2(\mathrm{~g} / \mathrm{kg}) \dagger$ & $15 \cdot 8$ & $19 \cdot 9$ \\
$\alpha-\mathrm{Linolenic} \mathrm{acid,} 18: 3(\mathrm{~g} / \mathrm{kg}) \dagger$ & $1 \cdot 29$ & $2 \cdot 17$ \\
PUFA:SFA ratio & $2 \cdot 34$ & $1 \cdot 70$ \\
Linoleic: $\alpha$-linolenic ratio & $12 \cdot 2$ & $9 \cdot 17$ \\
Protein $(\mathrm{g} / \mathrm{kg})$ & 163 & 133 \\
Water $(\mathrm{g} / \mathrm{kg})$ & $89 \cdot 1$ & $54 \cdot 6$ \\
Fibre $(\mathrm{g} / \mathrm{kg})$ & 156 & $60 \cdot 0$ \\
Ash (g/kg) & $46 \cdot 9$ & $30 \cdot 8$ \\
Cholesterol (mg/kg) & $69 \cdot 0$ & $26 \cdot 0 \ddagger$ \\
\hline
\end{tabular}

*The complete composition of vitamin and mineral mixtures of the SD (V10001 and S10026) and ND is available online (http://www.researchdiets.com and http:// www.panlab.com/en/).

The amounts of the five most abundant fatty acids found in both diets.

\# The information specified by the supplier of the SD indicates that the amount of cholesterol in this diet comes from lard. The SD has approximately $20 \mathrm{mg} / \mathrm{g}$ of lard, and a typical analysis of cholesterol in lard is $0.72 \mathrm{mg} / \mathrm{g}$ (Research Diets, Inc.). The cholesterol present in the ND comes from fish. Marine fish can contain between 100 and $1200 \mathrm{mg} / \mathrm{kg}$ body weight of cholesterol (information specified by Panlab).

was recorded every $2 \mathrm{~d}$, and daily food intake was recorded every 3-4 d during pregnancy and lactation. The morning on which newborn litters were found was designated as day 0 of lactation, and the litter size was adjusted to ten pups per dam. On day 21 of lactation, the dams were killed in ad libitum conditions under anaesthesia (pentobarbital sodium, $60 \mathrm{mg} / \mathrm{kg}$ body weight) within the first $2 \mathrm{~h}$ of the beginning of the light cycle. Blood was collected by cardiac puncture, and plasma obtained by centrifugation and stored at $-20^{\circ} \mathrm{C}$ until analysis. The liver was quickly removed following killing and weighed, and the hepatic index was calculated as the ratio of liver wet weight:body weight. Part of the liver from the left lobe was harvested and fixed with $4 \%$ paraformaldehyde in $0.1 \mathrm{M}$-phosphate buffer ( $\mathrm{pH} 7 \cdot 3$ ), while the remaining tissue was frozen in liquid $\mathrm{N}_{2}$ and stored at $-70^{\circ} \mathrm{C}$ until further analysis.

In addition, on day 21, two pups from each litter (one male and one female; the M-ND, F-ND, M-SD and F-SD groups) were killed by decapitation. Blood was collected and processed as described above.

Basal experiment. A total of twelve virgin rats were used for the control non-lactating groups, and assigned to a control non-lactating ND (ND-C group, $n$ 6) group or a control nonlactating SD (SD-C group, $n$ 6) group depending on the type of diet received during the $43 \mathrm{~d}$ period (ND or SD, respectively). Rats were fed ad libitum, and their body weight was recorded every $2 \mathrm{~d}$. On day 43 , the animals were killed in ad libitum conditions and under anaesthesia (pentobarbital sodium, $60 \mathrm{mg} / \mathrm{kg}$ body weight). Blood and liver samples were collected, processed and stored under the same conditions as described for the lactation experiment.

Pregnancy experiment. An extra group of rats fed SD from the first day of pregnancy to day 20 ( $n$ 9) was used to test whether the effects of this diet were specific for the lactation period or also occurred during pregnancy. Rats were fed ad libitum, and their body weight was recorded every $2 \mathrm{~d}$. On day 20 of pregnancy, the animals were killed in ad libitum conditions and under anaesthesia (pentobarbital sodium, $60 \mathrm{mg} / \mathrm{kg}$ body weight). Blood and liver samples were collected, processed and stored using the same conditions as described for the lactation experiment.

\section{Plasma analysis}

Enzymatic colorimetric kits were used to determine the levels of total cholesterol (reference no. 995280), TAG (reference no. 992320), glucose (reference no. 998282), glutamic-pyruvic transaminase (reference no. 998437), glutamic-oxaloacetic transaminase (GOT, reference no. 990823; QCA) and NEFA (reference no. 436-91995, 434-91795 and 270-77000; WAKO) in the plasma. Plasma insulin levels were measured using a mouse/rat insulin ELISA kit (reference no. EZRMI-13K; Millipore).

\section{Liver histology}

For morphological analysis, liver tissue samples ( $n$ 4-5 per group) were fixed by immersion in $4 \%$ paraformaldehyde in $0 \cdot 1 \mathrm{~m}$-phosphate buffer $(\mathrm{pH} 7 \cdot 3)$ at $4{ }^{\circ} \mathrm{C}$ for $24 \mathrm{~h}$, then washed with $0 \cdot 1 \mathrm{M}$-phosphate buffer $(\mathrm{pH} 7 \cdot 3)$, and dehydrated with a graded series of ethanol (50, 75, 96 and 100\%). After dehydration, tissues were cleared in xylene, infiltrated with paraplast embedding media (Sigma-Aldrich Química, S.A.) at $60^{\circ} \mathrm{C}$ overnight, and then embedded with fresh paraplast. Sections measuring $5 \mu \mathrm{m}$ were deparaffinised with xylene, hydrated with decreasing concentrations of ethanol and distilled water, then counterstained with haematoxylin and eosin, and observed with a Zeiss Axioskop 2 microscope equipped with an AxioCam ICc3 digital camera (Carl Zeiss, S.A.). Morphometric analysis was performed in the liver, as described previously ${ }^{(19)}$. Macrovesicular steatosis was evaluated according to Brunt et al. ${ }^{(27)}$ by analysing the entire histological section (approximate area $2 \mathrm{~cm}^{2}$ ) of the liver, estimating the percentage of the area covered by fat droplets and using a score graded from 0 to 3 ( 0 , null; 1 , when steatosis was detected in up to $30 \%$ of the area; 2, when steatosis was observed in between 30 and $60 \%$ of the area; 3, when steatosis was observed in more than $66 \%$ of the area).

\section{Extraction and quantification of total lipid content}

Lipids were extracted from the liver using the methods described by Hara \& Radin ${ }^{(28)}$ and Rodríguez-Sureda \& PeinadoOnsurbe $^{(29)}$ with modifications. Briefly, 60-100 mg of liver were mixed with $1 \mathrm{ml}$ hexane-isopropanol $(3: 2, \mathrm{v} / \mathrm{v})$. The tubes with the samples were gassed with $\mathrm{N}_{2}$ before being closed to minimise lipid oxidation and then left overnight under orbital agitation at room temperature and protected 
from light. The content of each tube was transferred into a new one and $0.3 \mathrm{ml}$ of $\mathrm{Na}_{2} \mathrm{SO}_{4}(0.47 \mathrm{M})$ were added. The tubes were mixed for $5 \mathrm{~min}$, left for $15 \mathrm{~min}$ in orbital agitation and centrifuged at $1000 \mathrm{~g}$ for $10 \mathrm{~min}$ at $4^{\circ} \mathrm{C}$. The upper phase containing lipids was dissolved in hexane and transferred to a clean, previously weighed glass tube. The hexane extract was then dried with $\mathrm{N}_{2}$ gas. Once the tube was dried, the percentage of lipids was determined as the weight difference between the tubes with lipid extracts and clean tubes, taking into account the initial amount of the tissue present. To evaluate TAG and cholesterol contents, the lipid extracts were dissolved in $1 \mathrm{ml}$ lipoprotein lipase buffer (28.75 mm-Pipes; $57.41 \mathrm{~mm}$ $\mathrm{MgCl}_{2} \cdot 6 \mathrm{H}_{2} \mathrm{O} ; 0.569 \mathrm{mg} / \mathrm{ml}$ of bovine serum albumin-NEFAfree) with $0 \cdot 1 \%$ SDS. Then, the samples were sonicated for $30 \mathrm{~s}$, and the tubes were left overnight in an orbital shaker at room temperature and protected from light. On the following day, the tubes were cold-sonicated with three pulses of $30 \mathrm{~s}$ each, and their TAG levels were measured immediately using total cholesterol (reference no. 995280) and TAG (reference no. 992320) enzymatic colorimetric kits (QCA)

\section{RNA extraction}

Total RNA from liver samples was extracted using the Tripure Reagent (Roche Diagnostics S.L.) and purified using the Qiagen RNeasy Mini Kit spin column (Izasa), according to the manufacturer's instructions. RNA yield was quantified in a NanoDrop ND-1000 spectrophotometer (NanoDrop Technologies), and integrity of RNA was confirmed using agarose gel electrophoresis.

\section{Real-time quantitative RT-PCR analysis}

Quantitative PCR was used to measure the mRNA expression levels of different genes involved in oxidative stress, metal toxicity, inflammation, fibrosis and apoptosis in the liver. Total RNA $(0.5 \mu \mathrm{g})$ was denatured and then reverse-transcribed to complementary DNA using MuLV RT (according to the procedure of Applied Biosystems) in a Perkin Elmer 9700 Thermal Cycler. For quantitative PCR analysis, the LightCycler 480 II System with the SYBR Green I Master Mix (Roche Diagnostics S.L.) was used. The PCR mixture for the different assays contained $2 \mu \mathrm{l}$ of sample complementary DNA (diluted 1:10), $0.55 \mu \mathrm{m}$ of each primer, $6 \mu \mathrm{l}$ of LightCycler 480 SYBR Green, and PCR-grade sterile water to a final volume of $11 \mu$ l. Thermocycling conditions were as follows: $95^{\circ} \mathrm{C}$ for $5 \mathrm{~min}$ for initial denaturation and activation of Taq polymerase, followed by forty-five thermal cycles of $95^{\circ} \mathrm{C}$ for $10 \mathrm{~s}, 60^{\circ} \mathrm{C}$ for $10 \mathrm{~s}$ and $72^{\circ} \mathrm{C}$ for $10 \mathrm{~s}$, with a ramping rate of $4 \cdot 4,2 \cdot 2$ and $4 \cdot 4^{\circ} \mathrm{C} / \mathrm{s}$, respectively. Fluorescence was measured once during each $72^{\circ} \mathrm{C}$ step. Following the amplification process, a melting curve analysis was performed by heating the plate at $95^{\circ} \mathrm{C}$ for $5 \mathrm{~s}$, incubating at $65^{\circ} \mathrm{C}$ for $1 \mathrm{~min}$ followed by a slow heating $\left(0 \cdot 11^{\circ} \mathrm{C} / \mathrm{s}\right)$ to $97^{\circ} \mathrm{C}$ (with five acquisitions per ${ }^{\circ} \mathrm{C}$ ). Fluorescence was monitored continuously during the melting experiment. Crossing point values $\left(C_{\mathrm{p}}\right)$ were determined using the LightCycler software (Roche Diagnostics S.L.). The primers for the different genes are described in online supplementary Table S1 and were obtained from Biomers.net. The relative expression of each mRNA was calculated as a percentage of the standard group using the $2^{-\Delta \Delta C_{\mathrm{t}}} \operatorname{method}^{(30)}$, with hypoxanthine guanine phosphoribosyl transferase (Hprt), peptidylprolyl isomerase A (Ppia) and transferrin receptor $(\operatorname{Tr} f \mathcal{C})$ as reference genes.

\section{Statistical analyses}

Data are expressed as means with their standard errors. The effect of diet on body weight and energy intake during pregnancy and lactation was assessed by repeated-measures ANOVA. Two-way ANOVA was used to determine the effects of different factors (diet, physiological status and sex) on plasma, liver parameters and gene expression. When one or more effects were statistically significant on two-way ANOVA, one-way ANOVA followed by Tukey's test was used to determine the differences between the means. Single comparisons between the groups were assessed by Student's $t$ test. All statistical analyses were performed using SPSS Statistics 18 (SPSS, Inc.), with the level of statistical significance being set at the $5 \%$ level (two-sided).

\section{Results}

\section{Body weight, and food and energy intake}

Fig. 1(A) shows the body weight of dams fed either the ND (ND-L group) or the SD (SD-L group) during pregnancy and lactation. The ND-L group had a higher body weight than the SD-L group (Fig. 1(A)). Specifically, ND-L dams increased their weight to 154 (sem 6) g from day 0 to day 21 of pregnancy, whereas SD-L dams increased to 120 (SEM 6) g. During lactation, body-weight gain was also higher in ND-L dams than in SD-L dams (28 (SEM 6) g $v,-7$ (SEM 3) g). This effect on body weight could be partially explained by the lower food and energy intake of SD-L dams during the experimental period, mainly during lactation (Fig. 1(B) and (C)). No changes were found either in body weight or energy intake during the experimental period between the control nonlactating groups fed either the control ND (ND-C) or the control SD (SD-C) (data not shown).

Fig. 2 shows total lipid consumption as well as intake of SFA, PUFA and MUFA during pregnancy and lactation. Furthermore, consumption of cholesterol and fibre during the two periods is also depicted. No changes were found on total fat consumption between the two groups of dams (Fig. 2(A)). In contrast, there were differences between the ND-L and the SD-L groups on SFA, PUFA, MUFA, cholesterol and fibre consumption during pregnancy and lactation. Specifically, consumption of SFA (Fig. 2(C)) and MUFA (Fig. 2(D)) was higher and the intake of PUFA (Fig. 2(E)), cholesterol (Fig. 2(B)) and fibre (Fig. 2(F)) was lower in the SD-L group than in the ND-L group.

\section{Plasma parameters, and weight and morphological features of the liver}

As expected, liver weight was higher and the plasma levels of insulin were lower in the ND-L group than in the ND-C group, 

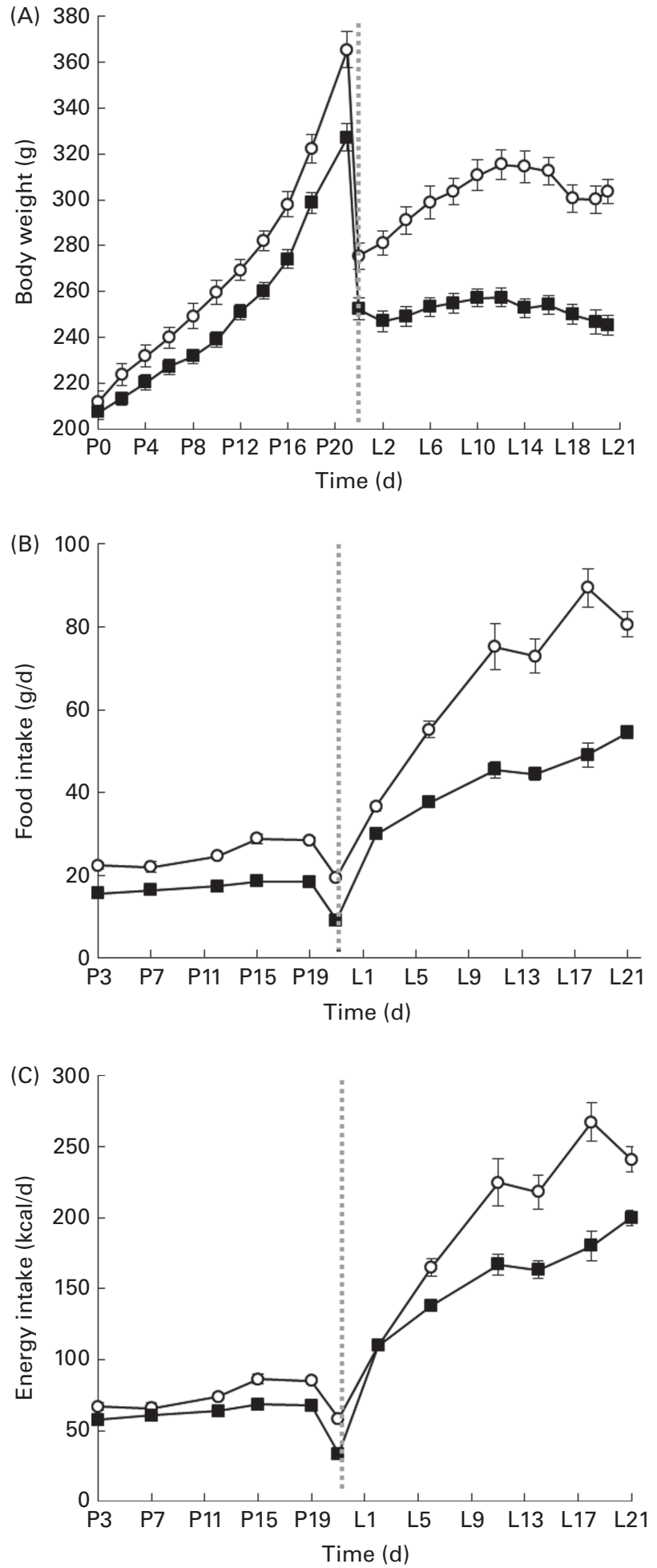

Fig. 1. (A) Body weight, (B) food intake and (C) energy intake of dams fed either a chow-based diet (ND) (ND-L group, -O-) or a semi-purified diet (SD) (SD-L group, - - ) during pregnancy and lactation. $P$ refers to pregnancy and $\mathrm{L}$ refers to lactation. Both the periods are delimited by vertical dotted lines. To calculate the energy intake, we assumed the energy content of the standard ND to be $1213 \mathrm{~kJ}(290 \mathrm{kcal}) / 100 \mathrm{~g}$ and that of the normal-fat SD to be $1536 \mathrm{~kJ}(367 \mathrm{kcal}) / 100 \mathrm{~g} .1 \mathrm{kcal}=4.184 \mathrm{~kJ}$. Values are means $(n 9)$, with their standard errors represented by vertical bars. Results of repeatedmeasures ANOVA: D, the effect of the type of diet; $T$, the effect of the time; $\mathrm{D} \times \mathrm{T}$, the interaction between the type of diet and time $(P<0.05)$. as a result of the endocrine regulation of metabolism that occurs during lactation ${ }^{(31)}$. In addition, as has been described previously, lactation led to a decrease in circulating TAG $^{(32,33)}$ and NEFA ${ }^{(32)}$ levels in ND-fed rats (Table 2). This metabolic pattern that occurs during lactation was impaired in the SD-L group; no change in insulin levels and higher levels of NEFA were found in comparison with the non-lactating SD-C group (Table 2).

The SD-L dams presented higher levels of circulating TAG and NEFA than the ND-L dams, while no changes were found in cholesterol plasma levels. Although no differences were observed in blood glucose levels, insulin levels were significantly higher in SD-L dams than in ND-L dams (Table 2). Histological analysis of the liver showed increased lipid deposition in the lactating dams of the SD-L group compared with those of the ND-L group (Fig. 3(A) and (B)). Specifically, the SD-L dams developed grade 3 macrovesicular steatosis, without apparent inflammation, fibrosis or necrosis (Fig. 3(B)). According to these observations, the SD-L rats had a higher hepatic index, as well as greater lipid, TAG and cholesterol concentrations, in the liver than the ND-L rats (Table 2). Moreover, plasma GOT and glutamic-pyruvic transaminase activities were significantly increased in SD-L dams compared with ND-L dams, indicating that fat accumulation in the liver induced by the SD during lactation caused liver damage (Table 2). Liver histology did not demonstrate changes in lipid deposition between the control non-lactating groups fed either the ND (ND-C group) or the SD (SD-C group) during the $43 \mathrm{~d}$ period (Fig. $3(\mathrm{C}$ ) and (D)). In addition, no changes were found in the liver and plasma parameters between these two groups (Table 2).

An extra group of rats fed the SD from the first day of pregnancy to day 20 was included to test whether the effects of this diet were specific to the lactation period or also occurred during pregnancy. The histological analysis of the liver from these rats did not show increased lipid deposition (data not shown), and, accordingly, the lipid levels in the liver from these rats $(34.9$ (SEM 1.3) $\mathrm{mg} / \mathrm{g}$ ) did not change significantly compared with the SD-C group and were lower than in the SD-L group.

\section{Characteristics of litters}

There were no significant differences in mean litter size $(12 \cdot 7$ (SEM 0.8) $v .12 .7$ (SEM 0.5)), mean number of male pups $(6 \cdot 1$ (SEM 1.2) v. $6 \cdot 0(\operatorname{SEm~} 0.7))$ and mean number of female pups (6.6 (SEM 0.8) v. 6.7 (SEM 0.8)) between litters born to the ND-L or SD-L dams. No changes in body weight were found between the male and female offspring of lactating rats fed the chow-based diet (M-ND and F-ND groups, respectively) or the semi-purified diet (M-SD and F-SD groups, respectively) at 1, 7 and $15 \mathrm{~d}$ of age, neither in males nor in females (data no shown). However, at $21 \mathrm{~d}$ of age, the M-ND and F-ND groups had a significantly higher body weight than the M-SD and F-SD groups (Table 3). In addition, the M-SD and F-SD groups showed increased levels of plasma TAG, cholesterol, NEFA and GOT compared with the M-ND and F-ND groups (Table 3). Neither glucose nor insulin blood levels changed between the groups, in both males and females. 

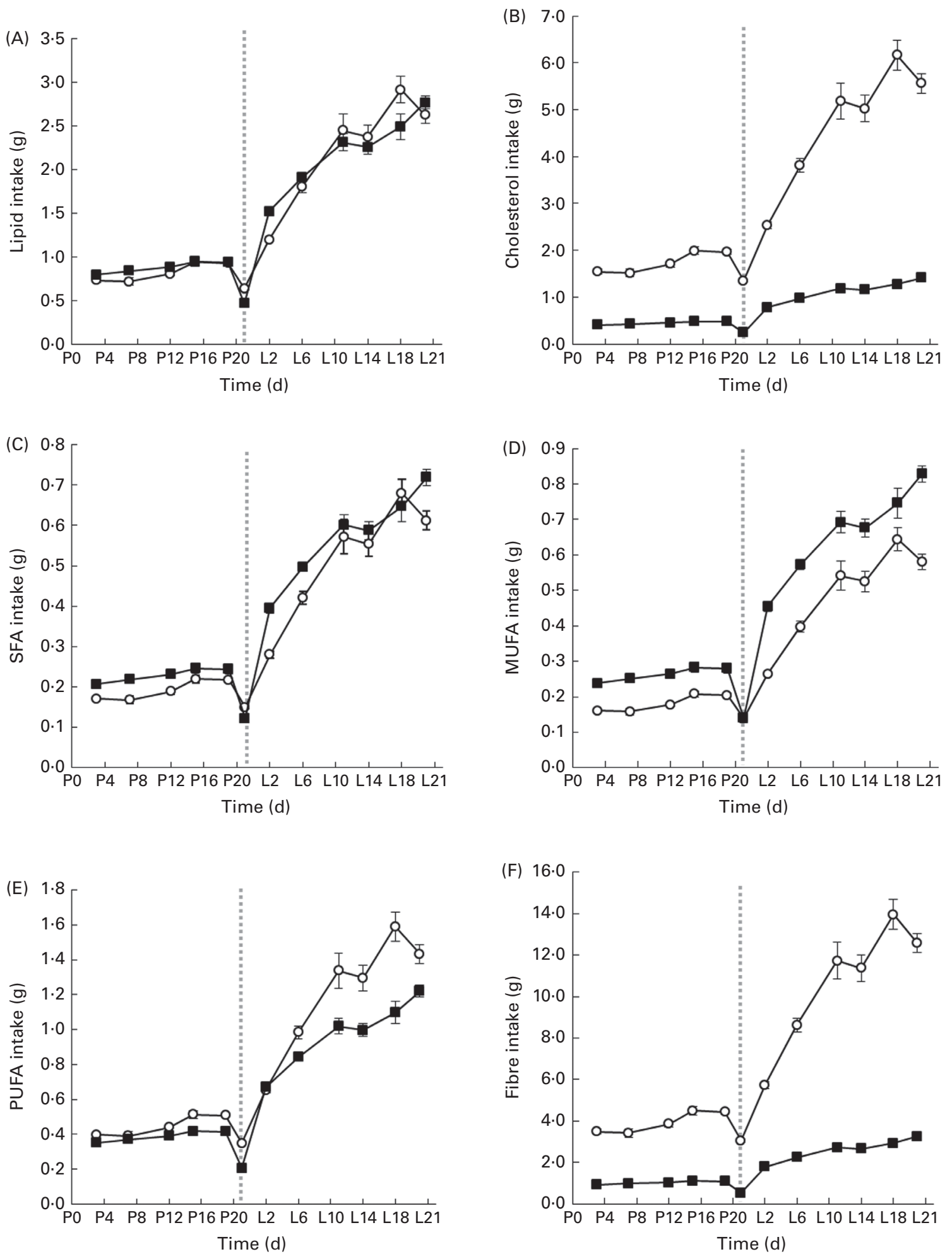

Fig. 2. (A) Lipid intake, (B) cholesterol intake, (C) SFA, (D) MUFA, (E) PUFA consumption and (F) fibre intake of lactating dams fed either a chow-based diet (ND) (ND-L group, -O-) or a semi-purified diet (SD) (SD-L group, -- ) during pregnancy and lactation. To calculate these parameters, we used the data about the composition of both diets shown in Table 1. P refers to pregnancy and $L$ refers to lactation. Both the periods are delimited by vertical dotted lines. Values are means $(n 9)$, with their standard errors represented by vertical bars. Results of repeated-measures ANOVA: D, effect of the type of diet; T, effect of time; D $\times$, interaction between the type of diet and time $(P<0.05)$. 
Table 2. Liver and plasma parameters of control or lactating rats fed the chow-based diet (ND) or the semi-purified diet (SD)*

(Mean values with their standard errors, $n 6-9$ )

\begin{tabular}{|c|c|c|c|c|c|c|c|c|c|}
\hline & \multicolumn{2}{|c|}{ ND-C group } & \multicolumn{2}{|c|}{ SD-C group } & \multicolumn{2}{|c|}{ ND-L group } & \multicolumn{2}{|c|}{ SD-L group } & \\
\hline & Mean & SEM & Mean & SEM & Mean & SEM & Mean & SEM & \\
\hline \multicolumn{10}{|l|}{ Liver parameters } \\
\hline Hepatic index (\%) & $3 \cdot 17^{a}$ & 0.09 & $3 \cdot 29^{a}$ & 0.12 & $4.54^{\mathrm{b}}$ & 0.07 & $6 \cdot 83^{\mathrm{C}}$ & 0.26 & $\mathrm{D}, \mathrm{P}, \mathrm{D} \times \mathrm{P} \dagger$ \\
\hline Lipids (mg/g) & $34 \cdot 8^{\mathrm{a}}$ & 1.6 & $35 \cdot 1^{\mathrm{a}}$ & $2 \cdot 3$ & $28 \cdot 6^{\mathrm{a}}$ & 0.8 & $255^{\mathrm{b}}$ & 19 & $D, P, D \times P$ \\
\hline $\mathrm{TAG}(\mathrm{mg} / \mathrm{g})$ & $2 \cdot 96^{\mathrm{a}}$ & 0.50 & $3.75^{\mathrm{a}}$ & 0.59 & $3.76^{\mathrm{a}}$ & 0.36 & $60 \cdot 28^{\mathrm{b}}$ & 9.50 & $D, P, D \times P$ \\
\hline Total cholesterol $(\mathrm{mg} / \mathrm{g})$ & $2 \cdot 00^{\mathrm{a}}$ & 0.04 & $1.89^{a}$ & 0.14 & $1.71^{a}$ & 0.12 & $6 \cdot 23^{\mathrm{b}}$ & $1 \cdot 12$ & $\mathrm{D}, \mathrm{P}, \mathrm{D} \times \mathrm{P}$ \\
\hline \multicolumn{10}{|l|}{ Plasma parameters } \\
\hline Glucose $(\mathrm{mmol} / \mathrm{l})$ & $5 \cdot 17^{\mathrm{a}}$ & 0.48 & $4.51^{\mathrm{a}, \mathrm{b}}$ & 0.32 & $3.85^{\mathrm{b}}$ & $0 \cdot 18$ & $4 \cdot 43^{\mathrm{a}, \mathrm{b}}$ & 0.21 & $P, D \times P$ \\
\hline Insulin (ng/ml) & $1.48^{\mathrm{a}}$ & 0.18 & $1.87^{\mathrm{a}}$ & 0.41 & $0.32^{\mathrm{b}}$ & 0.07 & $1.64^{\mathrm{a}}$ & 0.35 & $D, P, D \times P$ \\
\hline TAG $(\mathrm{mmol} / \mathrm{l})$ & $1.41^{\mathrm{a}}$ & 0.26 & $1.73^{\mathrm{a}}$ & $0 \cdot 10$ & $0.40^{\mathrm{b}}$ & 0.02 & $0.79^{\mathrm{c}}$ & 0.10 & $\mathrm{D}, \mathrm{P}$ \\
\hline Total cholesterol $(\mathrm{mmol} / \mathrm{l})$ & $1.66^{\mathrm{a}}$ & 0.07 & $1.80^{\mathrm{a}, \mathrm{b}}$ & $0 \cdot 10$ & $2 \cdot 18^{a, b}$ & 0.22 & $2 \cdot 48^{\mathrm{b}}$ & 0.19 & $\mathrm{P}$ \\
\hline NEFA $(\mathrm{mmol} / \mathrm{l})$ & $0.45^{\mathrm{a}}$ & 0.06 & $0.46^{\mathrm{a}}$ & 0.09 & $0 \cdot 10^{\mathrm{b}}$ & 0.02 & $0 \cdot 72^{\mathrm{a}}$ & 0.08 & $D, P, D \times P$ \\
\hline GOT (units/l) & $23 \cdot 7^{\mathrm{a}}$ & 1.3 & $28 \cdot 4^{\mathrm{a}}$ & $2 \cdot 0$ & $12 \cdot 6^{\mathrm{b}}$ & 0.9 & $50 \cdot 1^{\mathrm{c}}$ & 4.4 & $D, D \times P$ \\
\hline GPT (units/l) & $8 \cdot 19^{a}$ & 0.80 & $9 \cdot 38^{a}$ & 0.89 & $6 \cdot 57^{a}$ & 0.56 & $17 \cdot 5^{\mathrm{b}}$ & 1.0 & $D, P, D \times P$ \\
\hline
\end{tabular}

ND-C group, control non-lactating group fed the ND; SD-C group, control non-lactating group fed the SD; ND-L group, group fed the ND during pregnancy and lactation; SD-L group, group fed the SD during pregnancy and lactation; GOT, glutamic-oxaloacetic transaminase; GPT, glutamic-pyruvic transaminase.

${ }^{a, b, c}$ Mean values with unlike superscript letters were significantly different among the groups $(P<0.05$; one-way ANOVA and Tukey's post hoc comparison).

* Dams were fed the ND or SD (ND-L and SD-L groups, respectively) during gestation and lactation and killed on day 21 of lactation. Virgin rats were used for the control non-lactating groups and assigned to the ND-C or SD-C group depending on the diet received during the $43 \mathrm{~d}$ period.

$\dagger$ Results of two-way ANOVA: $\mathrm{D}$, the effect of the type of diet; $P$, the effect of physiological status; $\mathrm{D} \times \mathrm{P}$, the interaction between the type of diet and physiological status $(P<0.05)$.

\section{Gene expression in the liver}

To better characterise liver pathology associated with the intake of the SD during the lactation period, the mRNA levels of a set of genes related to inflammation, detoxification and oxidative stress responses, fibrosis and apoptosis were analysed in the liver of the ND-C, SD-C, ND-L and SD-L groups. With regard to the genes involved in inflammation, the intake of the SD during the lactation period highly increased the mRNA levels of Tnf $\alpha$ and monocyte chemoattractant protein 1 (Mcp1) compared with the ND group, while no changes were found in the expression of both genes among the other groups of rats (Fig. 4). One gene involved in protection against oxidative stress and metal toxicity (metallothionein 1, Mt1) was found to be sharply overexpressed in the liver of the ND-L group in comparison with the ND-C group as a result of normal physiological changes that occur during the lactation period ${ }^{(34)}$. However, this pattern of expression was not observed in the SD-L rats, in which Mt1 mRNA levels decreased in comparison with the other groups (Fig. 4), thereby suggesting an impairment of liver detoxification pathways in these lactating rats. No changes among the groups were found in the expression of glutathione $S$-transferase pi 1 (Gstp1) and haem oxygenase (decycling) (Hmox1), two genes that code for antioxidant enzymes. Liver steatosis that developed in the lactating dams fed the SD was also associated with a sharp increase in the mRNA levels of the fibrosis-related gene matrix metallopeptidase $9(M m p 9)^{(35)}$ (Fig. 4). In addition, the mRNA expression of caspase 3 (Casp3), a key gene involved in apoptosis ${ }^{(36)}$, tended to increase in the liver of the SD-L group compared with the ND-L group ( $P=0 \cdot 078$; Tukey's test) (Fig. 4).

\section{Discussion}

Various studies have shown that maternal sucrose intake during pregnancy and lactation can increase body weight and adiposity, and produce many metabolic disturbances in dams and their offspring (i.e. dyslipidaemia, fatty liver and insulin resistance $)^{(5-9)}$. Therefore, it is plausible to argue that substituting sucrose with starch may be beneficial when designing studies on pregnancy or metabolic imprinting to avoid metabolic alterations in both dams and their offspring. In contrast, although the use of SD among the scientific community has recently increased due to the low variability of this diet over time, ultimately leading to highly reproducible experiments ${ }^{(10)}$, the use of ND is still common among animal studies designed to examine the effect of nutritional interventions on metabolic imprinting in dams and their offspring $^{(17-22)}$. In the present study, we fed rats with two different diets, a SD and a ND, which can be considered as the standard, and have been used as control diets during pregnancy and lactation to assess the responses of lipid and glucose metabolism in dams and their offspring ${ }^{(12-22,26)}$.

Surprisingly, the present results point to important differences in the outcome of lipid metabolism between the two diets when administered during the lactation period. The main differences were found in the liver, where the SD caused grade 3 macrovesicular steatosis related to lipid accumulation. This result was reinforced by a higher hepatic index and greater lipid, TAG and cholesterol concentrations in the liver of lactating dams fed the SD. Moreover, we observed that rats fed the SD had higher gene expression levels of Tnf $\alpha$ and Mcp1 than those fed the ND, indicating a higher degree of hepatic inflammation. Furthermore, the 
(A)

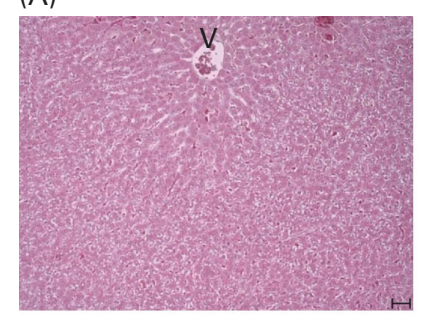

(C)

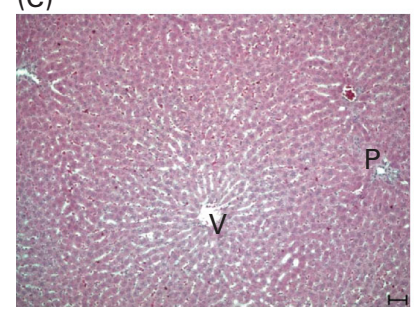

(B)

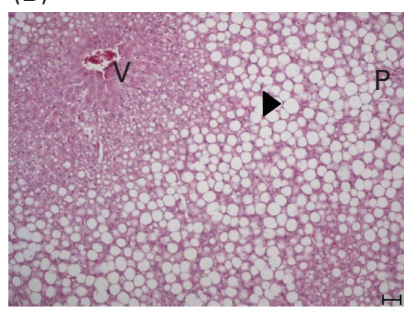

(D)

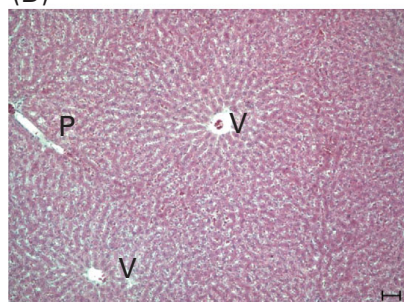

Fig. 3. Liver histology (haematoxylin and eosin staining, 10x) of lactating dams fed (A) a chow-based diet (ND) (ND-L group) or (B) a semi-purified diet (SD) (SD-L group) during pregnancy and lactation and killed on day 21 of lactation. Liver tissue sections from virgin rats used for the control non-lactating groups and assigned to a (C) control non-lactating ND group (ND-C group) or a (D) control non-lactating SD group (SD-C group), depending on the type of diet received during the $43 \mathrm{~d}$ period. V, central vein; $\mathrm{P}$, portal triad. (B) The triangle shows the lipid droplets present in the liver of rats in the SD-L group, and these animals developed macrovesicular steatosis. Scale bar, $50 \mu \mathrm{m}$.

decrease in the expression levels of the $M t 1$ gene and the increase in the expression levels of the Mmp9 gene in animals fed the SD suggest differences in oxidative stress response and a higher degree of hepatic fibrosis of these animals, respectively ${ }^{(35,36)}$. These alterations explain the increased levels of plasma glutamic-pyruvic transaminase and GOT, which, taken together, point to a significant degree of liver damage in SD-L rats. Furthermore, we observed other differences in the plasma parameters of both groups of lactating rats. Thus, despite the finding that body weight and energy intake were clearly lower in the SD-L group, probably due to different digestibilities and effective energy intakes between the diets ${ }^{(10)}$, insulin, TAG and NEFA levels were higher in these rats compared with the ND-L group. Due to the tight link between liver function, plasma lipids and insulin resistance ${ }^{(37,38)}$, it is plausible to hypothesise that the differences observed in the plasma between the two lactating groups are probably associated with altered liver metabolism. The results of the experiment using virgin rats suggest that the observed effects of the SD are probably due to interactions with pregnancy and/or lactation. In this experiment, virgin rats fed for the same period $(43 \mathrm{~d})$ with the SD showed no differences either in the liver or in plasma parameters compared with the ND-C group. Furthermore, the liver of rats fed the SD only during pregnancy (data not shown) did not present any evidence of fatty liver. These results reinforce the hypothesis that the reported negative effects of the SD on liver and lipid metabolism occur exclusively during the lactation period. It is important to note that the same SD used in the present study was also administered as a control diet to pregnant and lactating rats and their offspring to investigate whether a high-fat diet at different stages of development had adverse programming consequences on metabolic parameters and blood pressure ${ }^{(26)}$. In the present study, we clearly reported many effects on metabolic imprinting due to gestational, lactation and post-weaning diets. In addition, we compared the effects of both diets on the adiposity of dams at weaning. However, there are no data available for the effect of the diets on the liver weight and circulating parameters of dams ${ }^{(26)}$. In this sense, the present study highlights the importance of studying the impact of a nutritional intervention on the liver of dams used in experiments designed to assess the effects of maternal nutrition on offspring.

Differences, albeit mild, exist in the composition of both diets. There is a slight difference in the protein content between the two diets $(13.25 \%$ for the SD and $16.31 \%$ for the ND). Although the amount of protein present in the SD is lower than that recommended for the AIN-93G diet (17\%, taking into account that casein contains approximately $85 \%$ protein), the protein content of our diet matches with that recommended for the AIN-93M diet $(13-14 \%)^{(11)}$. Moreover, although some studies have shown that maternal dietary protein restriction during pregnancy ${ }^{(39)}$ and lactation ${ }^{(31)}$ is known

Table 3. Body weight and plasma parameters of 21-d-old male and female offspring from lactating rats fed the chow-based diet (ND) or the semi-purified diet (SD) for $43 \mathrm{~d}$

(Mean values with their standard errors, $n 9$ )

\begin{tabular}{|c|c|c|c|c|c|c|c|c|c|}
\hline & \multicolumn{2}{|c|}{ M-ND group } & \multicolumn{2}{|c|}{ M-SD group } & \multicolumn{2}{|c|}{ F-ND group } & \multicolumn{2}{|c|}{ F-SD group } & \\
\hline & Mean & SEM & Mean & SEM & Mean & SEM & Mean & SEM & \\
\hline Body weight (g) & $49 \cdot 5^{a}$ & 0.7 & $45 \cdot 4^{\mathrm{b}}$ & 0.7 & $50^{\mathrm{a}}$ & 0.7 & $44 \cdot 9^{\mathrm{b}}$ & 0.6 & $D^{\prime}$ \\
\hline Glucose (mmol/l) & $8 \cdot 17$ & 0.25 & 8.43 & 0.34 & $8 \cdot 12$ & 0.30 & $7 \cdot 64$ & 0.19 & \\
\hline Insulin (ng/ml) & 0.95 & 0.13 & 1.26 & 0.31 & 0.98 & $0 \cdot 12$ & 1.47 & 0.31 & \\
\hline TAG $(\mathrm{mmol} / \mathrm{l})$ & $1.75^{\mathrm{a}}$ & 0.18 & $2.96^{\mathrm{b}}$ & 0.27 & $1 \cdot 76^{\mathrm{a}}$ & 0.12 & $3 \cdot 12^{\mathrm{b}}$ & 0.27 & D \\
\hline Total cholesterol $(\mathrm{mmol} / \mathrm{l})$ & $3.33^{\mathrm{a}}$ & 0.18 & $3 \cdot 70^{a, b}$ & 0.11 & $3.45^{a, b}$ & $0 \cdot 16$ & $3.98^{\mathrm{b}}$ & 0.15 & $\mathrm{D}$ \\
\hline NEFA $(\mathrm{mmol} / \mathrm{l})$ & $0.56^{\mathrm{a}}$ & 0.05 & $0.89^{a, b}$ & 0.15 & $0.54^{\mathrm{a}}$ & 0.04 & $1.02^{\mathrm{b}}$ & 0.14 & D \\
\hline GOT (units/l) & $36 \cdot 9^{a}$ & 1.6 & $59 \cdot 5^{\mathrm{b}}$ & 1.6 & $37 \cdot 6^{a}$ & 2.4 & $58 \cdot 8^{\mathrm{b}}$ & $2 \cdot 4$ & $\mathrm{D}$ \\
\hline
\end{tabular}

M-ND group, male offspring of lactating rats fed for $43 \mathrm{~d}$ with the ND; M-SD group, male offspring of lactating rats fed for $43 \mathrm{~d}$ with the SD; F-ND group, female offspring of lactating rats fed for $43 d$ with the ND; F-SD group, female offspring of lactating rats fed for $43 d$ with the SD; GOT, glutamic-oxaloacetic transaminase.

a,b Mean values with unlike superscript letters were significantly different among the groups $(P<0.05$; one-way ANOVA and Tukey's post hoc comparison).

${ }^{*} \mathrm{D}$, the effect of the type of diet $(P<0.05$; two-way ANOVA). 


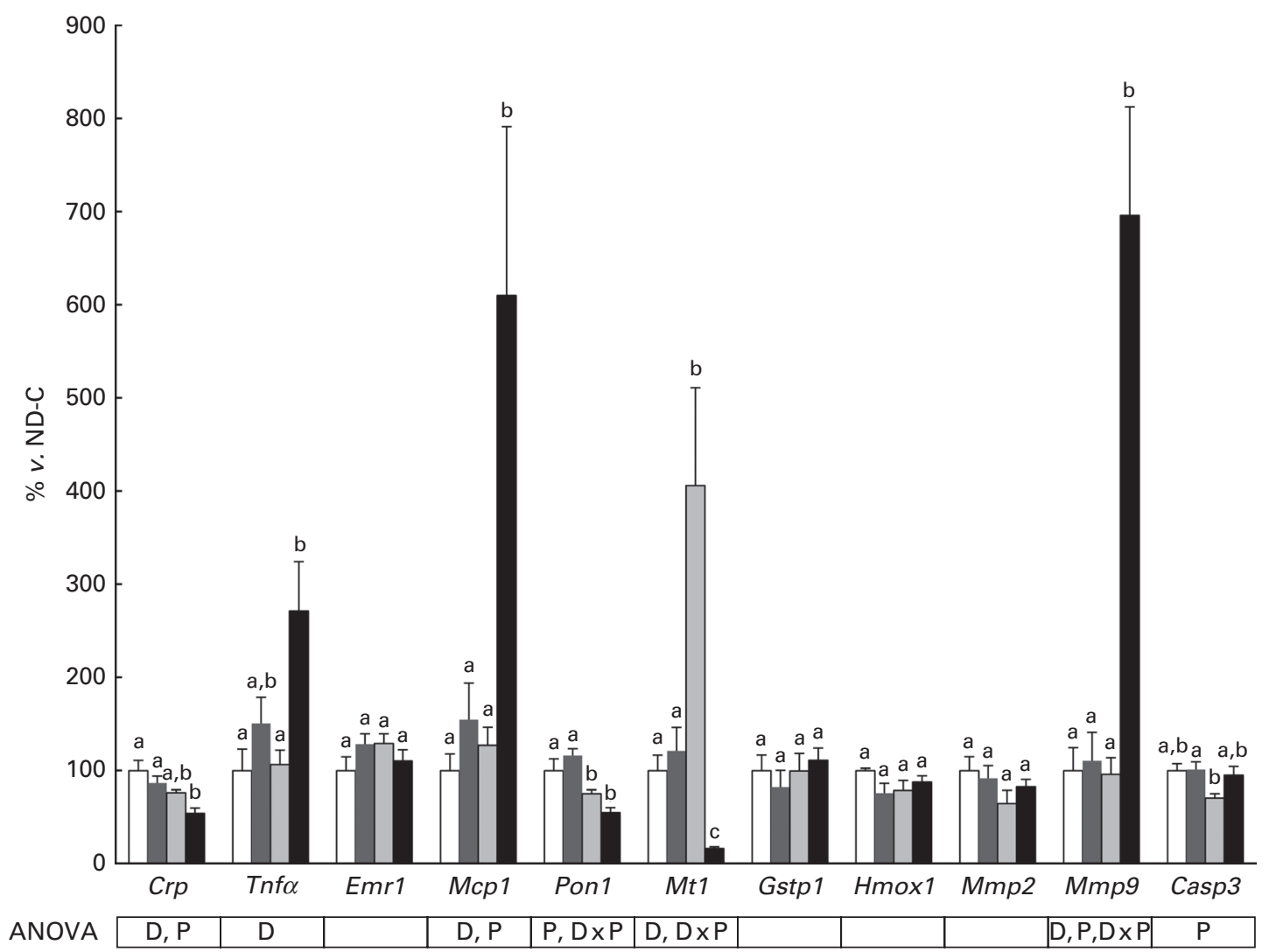

Fig. 4. mRNA expression levels of genes related to inflammation, macrophage infiltration, detoxification and oxidative stress responses, fibrosis and apoptosis in the liver of lactating and non-lactating rats. Lactating dams were fed a chow-based diet (ND) (ND-L group, $\square$ ) or a semi-purified diet (SD) (SD-L group, $\square$ ) during gestation and lactation, and killed on day 21 of lactation. Virgin rats were used for the control non-lactating groups and assigned to a control non-lactating ND group (ND-C group, $\square$ ) or a control non-lactating SD group (SD-C group, $\square$ ) depending on the type of diet received during the $43 \mathrm{~d}$ period. Values are means $(n 6-9)$, with their standard errors represented by vertical bars. Results of two-way ANOVA: D, effect of the type of diet; $P$, effect of physiological status; D $\times P$, interaction between the type of diet and physiological status $(P<0.05) .{ }^{\text {a,b,c }}$ Mean values with unlike letters were significantly different among the groups $(P<0.05$; one-way ANOVA and Tukey's post hoc comparison). Casp3, caspase 3; Crp, C-reactive protein; Emr1, egf-like module containing mucin-like, hormone receptorlike 1; Gstp1, glutathione $S$-transferase pi 1; Hmox1, haem oxygenase (decycling); Mcp1, monocyte chemoattractant protein 1; Mmp2, matrix metallopeptidase 2; Mmp9, matrix metallopeptidase 9; Mt1, metallothionein 1; Pon1, paraoxonase 1.

to alter the metabolism of dams, the levels of protein included in the diets to achieve these effects ( 8.5 and $6 \%$, respectively) were considerably lower than those used in the present study. Another difference between the two diets is that the SD contains $12 \%$ maltodextrin, a polysaccharide that is used as a food additive. However, it seems unlikely that metabolic disturbances found in the SD-L group are due to the presence of this nutrient, taking into account that maltodextrin is also included at very similar percentages (13\%) in the AIN-93G diet $^{(11)}$, and that dextrin-fed dams presented lower hepatic lipid levels and circulating TAG levels than sucrose-fed dams ${ }^{(6)}$. In contrast, it could be speculated that the higher fibre content of the ND could underlie the differences found between the two diets because fibre intake has been described to be beneficial against the development of a fatty liver ${ }^{(40)}$ and in the improvement of the lipid profile ${ }^{(41,42)}$. Furthermore, although we have only information about the total amount of fibre present in both diets, the presumable different source of fibre between the SD and ND diets (cellulose in the SD and cellulose, hemicellulose and lignin mainly coming from grain cereals and bran in the ND) could also account for the observed results, since different studies have reported that consumption of insoluble fibres obtained from vegetables, nuts and fruits produces a decrease in circulating cholesterol and TAG levels when compared with cellulose present in SD diets ${ }^{(43-45)}$. However, it is important to take into account that the source of fibre present in the SD is similar to that recommended for both AIN-93M and AIN-93G diets (cellulose), and that the amount included matches with that recommended for both AIN diets to a larger extent. For these reasons, it is unlikely that the differences in the source and content of fibre between the SD and the ND can be responsible of the observed effects.

Another difference between the two diets used in the present study is the fat and cholesterol content. The SD contains more fat, and although it cannot be considered a high-fat diet, induction of fatty liver in the SD-L group could be related to increased hepatic load of $\mathrm{fat}^{(37)}$. Nevertheless, the SD-L group presents a clearly lower food intake than the ND-L group, resulting in a similar net consumption of fat between the two groups. The predominant fatty acids are the same in both the diets, but their proportions differ, resulting in a different intake of SFA, MUFA and PUFA between the ND and SD groups. Very recently, a study conducted in human subjects 
has shown that overfeeding of fat present a very different outcome depending on the type of fatty acids administered ${ }^{(46)}$. Thus, individuals consuming an excess of fats in the form of PUFA gained weight due to increased lean mass and fat mass in a 1:1 proportion. Instead, in individuals consuming the same amount of fats in the form of SFA, the body-weight gain was due to increased lean mass and fat mass in a 1:4 proportion. In these individuals, the increase in liver ectopic fat was proportional to plasma SFA concentration. In the present study, the SD contained slightly more SFA and less PUFA. In virgin or pregnant rats, these differences might not be relevant, since the net intake of fats is low and insufficient to cause dyslipidaemia or fatty liver in the short term. On the contrary, lactating rats present an evident hyperphagia, and therefore, the differences in fatty acid consumption are exacerbated. In this case, lower PUFA and higher SFA intake of the SD-L group could explain the differential effects in lipid metabolism and the increased ectopic fat accumulation. Although this is a plausible explanation, it is worth noting that we do not have data regarding fat digestibility and absorption in these rats, and we cannot know whether increased SFA intake in the SD-L group results in an effective increase in SFA load into the liver. In relation to the current recommendations for laboratory rodents, the AIN-93 formulation suggested the use of soyabean oil instead of maize oil in order to meet the requirements of linoleic acid, resulting in a PUFA:SFA ratio about 4. Although, in the present study, the PUFA:SFA ratio is approximately 2 for both the diets, it is well accepted that a PUFA:SFA ratio of approximately 2 can be considered as a healthy dietary fatty acid profile for laboratory rodents ${ }^{(47)}$ and is, therefore, unlikely the cause of the metabolic differences among the ND-L and SD-L groups.

Independently of the PUFA:SFA ratio, increasing MUFA intake is translated into higher plasma and liver lipids in rats $^{(48)}$. The slightly higher MUFA content of the SD did not result in deleterious effects in virgin and pregnant rats; however, again, the higher food intake of lactating dams was translated into important differences in MUFA consumption between the ND-L and SD-L rats. Thus, the higher MUFA intake of the SD-L group could be under the observed effects in lipid metabolism. Another factor that has an impact on the correct maintenance of the lipid metabolism of the rat is the linoleic: $\alpha$-linolenic (or the $n-6: n-3$ fatty acids) ratio. In the AIN-93 diet, this ratio is $6^{(49)}$, and in our case, the ratio is close to 9 for the SD and 12 for the ND. Since the SD ratio falls between the two AIN-93 diets and the ND diet, it could be suggested that linoleic and $\alpha$-linolenic content of the $\mathrm{SD}$ is not responsible of the deleterious effects observed in the SD-L group.

Cholesterol has also been shown to be relevant in the generation of fatty liver ${ }^{(37)}$. However, in contrast with fat composition, the amount of cholesterol in the SD is less than one half that of the ND. Together with diet composition, the lower food intake of SD-L rats results in a lower cholesterol intake in $\mathrm{SD}-\mathrm{L}$ rats compared with ND-L rats. Therefore, it is plausible to hypothesise that the increased levels of plasma and liver cholesterol are due to differences in lipid metabolism rather than to increased cholesterol intake.
Another possibility to explain the contrasting effects of the ND and SD on lactation could be the differences in micronutrient content, or ratios, among diets. It is worth noting that the micronutrient composition of our SD is not the one proposed by the AIN-93G standard. However, our diet included the same mineral and vitamin mixes as other SD used in studies during pregnancy and lactation, and most importantly, we did not report the deleterious effects on dams as a consequence of this diet ${ }^{(12-16)}$. Nevertheless, slight excess or deficiency of a given micronutrient could have been exacerbated by the higher metabolic demand during the lactation period. Finally, another explanation could be the presence of bioactive compounds in the ND but not in the SD, such as phyto-oestrogens, which may affect different hormonal signalling processes ${ }^{(50,51)}$. It could be speculated that the usual hyperphagia directed to increase the energy expenditure for milk production ${ }^{(52)}$ might involve a higher intake of those compounds that can promote or prevent hepatic alterations. This may also explain the lack of any adverse effects in virgin or pregnant rats fed the SD because these animals maintain a normal food intake.

Overall, it is difficult to link the profound differences observed between the ND-L and SD-L dams and their respective offspring to differences in a single component of the diet. Instead, the sum of the apparently slight differences in protein content, fatty acid profile or the presence of fibre, among others, might be responsible of the evident differences in the lipid metabolism of lactating mothers and their offspring.

The mechanisms leading to metabolic imprinting, such as DNA methylation ${ }^{(1,2)}$, may be affected in several ways by maternal diet. It has been shown that different sources of energy (either fat or carbohydrates) may affect the sex ratio of pups ${ }^{(16)}$. The offspring of dams that were fed high-fat diets presented increased adiposity and altered levels of insulin and leptin ${ }^{(26)}$. Protein restriction has been reported to induce epigenetic changes in the liver and in fetal brain composition $^{(39,53)}$. Additionally, even the ratio of linoleic: $\alpha$-linolenic acid can affect the levels of leptin in the offspring $^{(54)}$. Moreover, beyond the effects of maternal diet, it has also been shown that metabolic alterations of dams can result in altered states of the offspring. Thus, maternal inflammation derived from obesity can lead to changes in the brain of the offspring, resulting in hippocampal inflammation and changes in spatial learning and anxiety ${ }^{(14)}$, and gestational insulin resistance in insulin receptor substrate-1 haploinsufficient mice results in glucose intolerance, insulin resistance and lipid metabolism alterations in the offspring ${ }^{(55)}$. In agreement with these findings, the present results show differences between the two diets in the effect on offspring metabolism, such as increased levels of TAG, cholesterol, NEFA and GOT, a plasma marker of hepatic injury, in the offspring of the SD-L group, even when dams are fed a widely used standard control diet.

In conclusion, the present results show that two different diets that have been used as control diets in metabolic imprinting experiments present very different outcomes. Despite both diets have no differential metabolic effects in virgin and gestating rats, the interaction with the lactation period is completely 
different and, importantly, can influence differently the metabolic parameters of the offspring. SD are highly recommended due to the reproducibility and the control over nutritional parameters that can influence different mechanisms of imprinting such as hormonal status and presence of a bioactive compound. Nevertheless, the present results suggest that assessment of the metabolic state of dams, and not only of their offspring, might be relevant when interpreting the results of metabolic programming studies. Moreover, it is tempting to extrapolate the results of the present study to human nutrition because the metabolic imprinting phenomenon has been suggested by different authors as an innovative strategy to prevent the high prevalence of obesity in developed and developing countries ${ }^{(56,57)}$. The present results suggest that small changes within a normal diet might result in profound changes in the offspring. Therefore, it is plausible to speculate that nutritional recommendations for the lactation period can still be optimised in order to make this strategy feasible, although more studies are needed in order to achieve this ambitious goal.

\section{Supplementary material}

To view supplementary material for this article, please visit http://dx.doi.org/10.1017/S0007114514004358

\section{Acknowledgements}

The authors gratefully acknowledge Vanessa Grifoll, laboratory technician, for assistance.

The present study was funded by ACC1Ó (TECRD121-0005) and by the Spanish Ministry of Economy and Competitiveness (MECC) (AGL2010-19585, IMPROBES Project).

The authors' contributions are as follows: J. M. d. B., A. Ca., A. P., L. A. and A. Cr. conceived and designed the experiments; J. M. d. B., A. Ca., E. C., A. A.-A. and A. Cr. performed the experiments and analysed the data; J. M. d. B., A. Ca., A. P., L. A. and A. Cr. wrote the paper.

The authors declare that they have no conflict of interest.

\section{References}

1. Sullivan EL \& Grove KL (2010) Metabolic imprinting in obesity. Forum Nutr 63, 186-194.

2. Canani RB, Costanzo MD, Leone L, et al. (2011) Epigenetic mechanisms elicited by nutrition in early life. Nutr Res Rev 24, 198-205.

3. Christian P \& Stewart CP (2010) Maternal micronutrient deficiency, fetal development, and the risk of chronic disease. J Nutr 140, 437-445.

4. Jackson AA, Dunn RL, Marchand MC, et al. (2002) Increased systolic blood pressure in rats induced by a maternal low-protein diet is reversed by dietary supplementation with glycine. Clin Sci 103, 633-639.

5. Ghusain-Choueiri AA \& Rath EA (1995) Effect of carbohydrate source on lipid metabolism in lactating mice and on pup development. Br J Nutr 74, 821-831.

6. Oliveros L, Giménez I \& Giménez MS (1995) Effect of sucrose feeding during pregnancy on rat maternal and fetal liver lipid and glycogen metabolism. Biosci Biotechnol Biochem 59, 412-416.

7. Sedová L, Seda O, Kazdová L, et al. (2007) Sucrose feeding during pregnancy and lactation elicits distinct metabolic response in offspring of an inbred genetic model of metabolic syndrome. Am J Physiol Endocrinol Metab 292, E1318-E1324.

8. Bourne AR, Richardson DP, Bruckdorfer KR, et al. (1975) Some effects of different dietary carbohydrates on pregnancy and lactation in rats. Nutr Metab 19, 73-90.

9. Oliveros L, Callegari E \& Giménez MS (1997) Effect of sucrose feeding on glucose tolerance and their relationship with lipid metabolism in maternal and fetal livers in rat. Arch Physiol Biochem 105, 640-644.

10. Hoevenaars FPM, van Schothorst EM, Horakova O, et al. (2012) BIOCLAIMS standard diet (BIOsd): a reference diet for nutritional physiology. Genes Nutr 7, 399-404.

11. Reeves PG, Nielsen FH \& Fahey GC (1993) AIN-93 purified diets for laboratory rodents: final report of the American Institute of Nutrition ad hoc writing committee on the reformulation of the AIN-76A rodent diet. J Nutr 123, 1939-1951.

12. Hernandez LL, Grayson BE, Yadav E, et al. (2012) High fat diet alters lactation outcomes: possible involvement of inflammatory and serotonergic pathways. PLOS ONE 7, e32598.

13. Page KC, Malik RE, Ripple JA, et al. (2009) Maternal and postweaning diet interaction alters hypothalamic gene expression and modulates response to a high-fat diet in male offspring. Am J Physiol Regul Integr Comp Physiol 297, R1049-R1057.

14. Bilbo SD \& Tsang V (2010) Enduring consequences of maternal obesity for brain inflammation and behavior of offspring. FASEB J 24, 2104-2115.

15. Gallou-Kabani C, Vige A, Gross M-S, et al. (2006) Resistance to high-fat diet in the female progeny of obese mice fed a control diet during the periconceptual, gestation, and lactation periods. Am J Physiol Endocrinol Metab 292, E1095-E1100.

16. Rosenfeld CS, Grimm KM, Livingston KA, et al. (2003) Striking variation in the sex ratio of pups born to mice according to whether maternal diet is high in fat or carbohydrate. Proc Natl Acad Sci U S A 100, 4628-4632.

17. Sánchez J, Priego T, Palou M, et al. (2008) Oral supplementation with physiological doses of leptin during lactation in rats improves insulin sensitivity and affects food preferences later in life. Endocrinology 149, 733-740.

18. García-Peláez B, Vilà R \& Remesar X (2008) Treatment of pregnant rats with oleoyl-estrone slows down pup fat deposition after weaning. Reprod Biol Endocrinol 6, 23.

19. García AP, Palou M, Sánchez J, et al. (2011) Moderate caloric restriction during gestation in rats alters adipose tissue sympathetic innervation and later adiposity in offspring. PLOS One 6, e17313.

20. McMullin TS, Lowe ER, Bartels MJ, et al. (2008) Dynamic changes in lipids and proteins of maternal, fetal, and pup blood and milk during perinatal development in CD and Wistar rats. Toxicol Sci 105, 260-274.

21. Picó C, Oliver P, Sánchez J, et al. (2007) The intake of physiological doses of leptin during lactation in rats prevents obesity in later life. Int J Obes 31, 1199-1209.

22. Palou M, Priego T, Sánchez J, et al. (2010) Moderate caloric restriction in lactating rats protects offspring against obesity and insulin resistance in later life. Endocrinology 151, 1030-1041.

23. Arola-Arnal A, Oms-Oliu G, Crescenti A, et al. (2013) Distribution of grape seed flavanols and their metabolites 
in pregnant rats and their fetuses. Mol Nutr Food Res 57, $1741-1752$

24. Emiliano AF, de Cavalho LC, da Silva Cristino Cordeiro V, et al. (2011) Metabolic disorders and oxidative stress programming in offspring of rats fed a high-fat diet during lactation: effects of a vinifera grape skin (ACH09) extract. J Cardiovasc Pharmacol 58, 319-328.

25. Mukai Y, Sun Y \& Sato S (2013) Azuki bean polyphenols intake during lactation upregulate AMPK in male rat offspring exposed to fetal malnutrition. Nutrition 29, 291-297.

26. Mitra A, Alvers KM, Crump EM, et al. (2009) Effect of high-fat diet during gestation, lactation, or postweaning on physiological and behavioral indexes in borderline hypertensive rats. Am J Physiol 296, R20-R28.

27. Brunt EM, Janney CG, Di Bisceglie AM, et al. (1999) Nonalcoholic steatohepatitis: a proposal for grading and staging the histological lesions. Am J Gastroenterol 94, 2467-2474.

28. Hara A \& Radin NS (1978) Lipid extraction of tissues with a low-toxicity solvent. Anal Biochem 90, 420-426.

29. Rodríguez-Sureda V \& Peinado-Onsurbe J (2005) A procedure for measuring triacylglyceride and cholesterol content using a small amount of tissue. Anal Biochem 343, $277-282$.

30. Livak KJ \& Schmittgen TD (2001) Analysis of relative gene expression data using real-time quantitative PCR and the 2(-Delta Delta C(T)) method. Methods 25, 402-408.

31. Moretto VL, Ballen MO, Gonçalves TSS, et al. (2011) Lowprotein diet during lactation and maternal metabolism in rats. ISRN Obstet Gynecol 2011, 876502-876508.

32. Gutgesell A, Ringseis R, Schmidt E, et al. (2009) Downregulation of peroxisome proliferator-activated receptor alpha and its coactivators in liver and skeletal muscle mediates the metabolic adaptations during lactation in mice. $J \mathrm{Mol}$ Endocrinol 43, 241-250.

33. LaBorde JB, Wall KS, Bolon B, et al. (1999) Haematology and serum chemistry parameters of the pregnant rat. Lab Anim 33, 275-287.

34. Solaiman D, Jonah MM, Miyazaki W, et al. (2001) Increased metallothionein in mouse liver, kidneys, and duodenum during lactation. Toxicol Sci 60, 184-192.

35. Yang SF, Tseng JK, Chang YY, et al. (2009) Flaxseed oil attenuates nonalcoholic fatty liver of hyperlipidemic hamsters. J Agric Food Chem 57, 5078-5083.

36. Kirpich IA, Gobejishvili LN, Bon M, et al. (2011) Integrated hepatic transcriptome and proteome analysis of mice with high-fat diet-induced nonalcoholic fatty liver disease. $J$ Nutr Biochem 22, 38-45.

37. Enjoji M, Yasutake K, Kohjima M, et al. (2012) Nutrition and nonalcoholic fatty liver disease: the significance of cholesterol. Int J Hepatol 2012, 925807-925812.

38. Farese RV, Zechner R, Newgard CB, et al. (2012) The problem of establishing relationships between hepatic steatosis and hepatic insulin resistance. Cell Metab 15, 570-573.

39. Torres N, Bautista CJ, Tovar AR, et al. (2010) Protein restriction during pregnancy affects maternal liver lipid metabolism and fetal brain lipid composition in the rat. Am J Physiol Endocrinol Metab 298, E270-E277.

40. Zelber-Sagi S, Ratziu V \& Oren R (2011) Nutrition and physical activity in NAFLD: an overview of the epidemiological evidence. World J Gastroenterol 17, 3377-3389.

41. Chandalia M, Carg A, Lutjohann D, et al. (2000) Beneficial effects of high dietary fiber intake in patients with type 2 diabetes mellitus. $N$ Engl J Med 342, 1392-1398.
42. Slavin J (2013) Fiber and prebiotics: mechanisms and health benefits. Nutrients 5, 1417-1435.

43. Caimari A, Puiggros F, Suarez M, et al. (2015) The intake of a hazelnut skin extract improves the plasma lipid profile and reduces the lithocholic/deoxycholic bile acid fecal ratio, a risk factor for colon cancer, in hamsters fed a high-fat diet. Food Chem 167, 138-144.

44. Lecumberri E, Goya L, Mateos R, et al. (2007) A diet rich in dietary fiber from cocoa improves lipid profile and reduces malondialdehyde in hypercholesterolemic rats. Nutrition 23, 332-341.

45. Chau CF \& Huang YL (2005) Effects of the insoluble fiber derived from Passiflora edulis seed on plasma and hepatic lipids and fecal output. Mol Nutr Food Res 49, 786-790.

46. Rosqvist F, Iggman D, Kullberg J, et al. (2014) Overfeeding polyunsaturated and saturated fat causes distinct effects on liver and visceral fat accumulation in humans. Diabetes $\mathbf{6 3}$ $2356-2368$.

47. Lee JH, Fukumoto M, Nishida H, et al. (1989) The interrelated effects of $n-6 / n-3$ and polyunsaturated/saturated ratios of dietary fats on the regulation of lipid metabolism in rats. J Nutr 119, 1893-1899.

48. Chang NW \& Huang PC (1998) Effects of the ratio of polyunsaturated and monounsaturated fatty acid to saturated fatty acid on rat plasma and liver lipid concentrations. Lipids $\mathbf{3 3}$, 481-487.

49. Bourre JM, Francois A, Youyou O, et al. (1989) The effects of dietary alpha-linolenic acid on the composition of nerve membranes, enzymatic activity, amplitude of electrophysiological parameters, resistance to poisons and performance of learning tasks in rats. J Nutr 119, 1880-1892.

50. Brown NM \& Setchell KD (2001) Animal models impacted by phytoestrogens in commercial chow: implications for pathways influenced by hormones. Lab Invest 81, 735-747.

51. Akingbemi BT, Braden TD, Kemppainen BW, et al. (2007) Exposure to phytoestrogens in the perinatal period affects androgen secretion by testicular Leydig cells in the adult rat. Endocrinology 148, 4475-4488.

52. Grigor MR, Allan JE, Carrington JM, et al. (1987) Effect of dietary protein and food restriction on milk production and composition, maternal tissues and enzymes in lactating rats. J Nutr 117, 1247-1258.

53. Lillycrop KA, Phillips ES, Jackson AA, et al. (2005) Dietary protein restriction of pregnant rats induces and folic acid supplementation prevents epigenetic modification of hepatic gene expression in the offspring. J Nutr 135, 1382-1386.

54. Korotkova M, Gabrielsson B, Lönn M, et al. (2002) Leptin levels in rat offspring are modified by the ratio of linoleic to alpha-linolenic acid in the maternal diet. J Lipid Res $\mathbf{4 3}$, 1743-1749.

55. Isganaitis E, Melissa W, Huijuan M, et al. (2014) Developmental programming by maternal insulin resistance hyperinsulinemia, glucose intolerance, and dysregulated lipid metabolism in male offspring of insulin-resistant mice. Diabetes 63, 688-700.

56. Tang W \& Ho S (2007) Epigenetic reprogramming and imprinting in origins of disease. Rev Endocr Metab Disord 8, 173-182.

57. Levin BE (2006) Metabolic imprinting: critical impact of the perinatal environment on the regulation of energy homeostasis. Philos Trans R Soc Lond B Biol Sci 361, 1107-1121. 